\section{Spectroscopic standard could help revolutionise the analysis of plastics}

Plastic pollution is an environmental catastrophe in progress. 32 percent of the plastic we use escapes into the environment and only nine percent currently gets recycled. Fugitive plastic products often undergo various forms of environmental degradation, which lead to the formation of microplastics. British company Polymateria have developed escape the waste stream. This fresh approach is also a rigorous one. The team at Polymateria noted that convention al methods to dete. carbonyl index, a measure of plastic durability or decay, lacked coherence: there was no agreement in the industry about what type of analysis works best. Their proposal - to standardise the use of Specific Area Under Band (SAUB) analysis to determine carbonyl index - has now been taken up by the American Society for Testing and Materials, as well as the British Standards Institute, as the preferred way to assess plastic transformation.

381 million tonnes of plastic Plastic packaging is the primary 381 were created in 2015: driving factor for plastic generation, 30 that's roughly two-thirds World in Dat). Syman population (Our than a centry old but shastics are lass than a century old but, since the mass ${ }^{\prime} 50$ s, 5800 million tonnes of the plastic produced has been disposed of only nin percent of which has been rect, only nine staggering 32 percent estimated to have escaped into the natural environment. accounting for almost 50 percent of the plastic made worldwide. In this context, it is hard to ignore the reason plastic is so popular: it's ch versatle, mouldable, light, and Plastic can sometimt break down easly. conveniently and for the same reason can often be disposed of without consideration.

\section{THE MICROPLASTIC}

MEGA-PROBLEM

But

the environment, and why

Global annual production of plastic waste continues at a monumental scale and increases every year. Meanwhile, global waste management streams fail to keep pace with the growing global population and increased consumerism. Each year, megaton of synthetic plastics leak into the environment, around 10 megatons of which enters the oceans. The rest, a staggering 40 megatons, remains on land and is considered 'terrestrial fugitive plastic'.

As these plastic products break down they form microscopic fragments known risk is that they are key environmental Thisk is that they are biologically inert. in the natural environment.

We know that we need to limit the amount of plastic and microplastic pollution, however it is not as simple as turning off the 'plastic tap'. In order to enable the removal of standard plastic products from production, sustainable, suitable replacements must be secured as plastics have a plethora of vital uses. These include: single-use sterile medica equipment, to reduce the risk of crosscontamination in hospitals; hygienic food transport and storage solutions, to reduce food waste; lightweight transport and efficient insulation, to reduce

Givi-tul based ensergy consumption. Given the curent scale of plasticuse it is

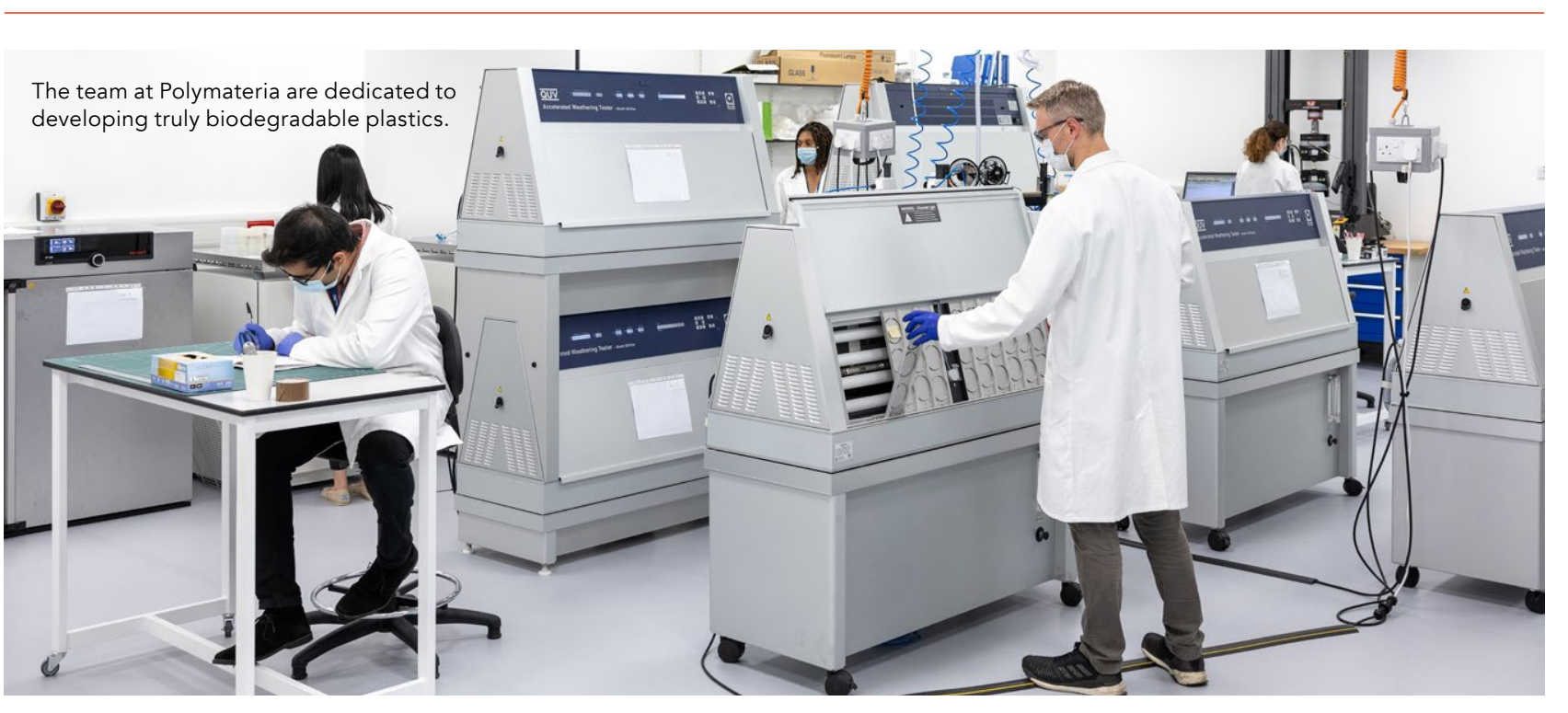

environment. So, what can be done to tackle the problem?

\section{POLYMATERIA'S SOLUTION}

British company Polymateria are

workics that impart biodegradability

should they become plastic pollution

on land in our environment".

SETTING NEW STANDARDS Plastic degradation is caused by manufacturing of products and agion to use, especially in the case of outdoor to slications. The ability to predict the lifespan of a plastic product, whilst it is in use, is of critical importance to both plastic manufacturers and consumers. Furthermore, the potential to prove the durability of a material under natural

Polymateria have designed an 'innovation inside'
and manufacture technologies for various types of common plastic products that are designed to impart time-delayed biodegradability. Their technology can be used plastics, like pim is to ensure that their plastic products becom biodegradable wax rather than fragmenting into microplastics, should they slip through the net of waste management systems and end up in the environment, thereby avoiding further contribution to the problem of plastic pollution.

To create this new technology, the team of scientists at Polymateria have had to revolutionise scientific thinking on the breakdown process of different plastics under different environmental conditions. This has meant going beyond standardised testing practices and has led to the development of plastic analysis.
Problematically, many studies have stated carbonyl index values with wildly different values, making comparisons meaningless.

environmental stresses (sunlight, wind, rain, etc.) is essential to aid and advance inderstanding and development that do not add to plastic pollution bioaccumulation and consequent environmental damage.

Infrared methods are commonly used to test plastics, like polypropylene and polyethylene, and provide a value referred to as the carbonyl index (CI), on the breakdown of a material. To date,

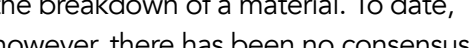
regarding which of the multiple possible techniques should be used to calculate as the definitive technique. By comparing and contrasting each technique using the same material, the team at Polymateria found that the different methods of
Clvalues; with so much inconsistency

\section{COMPARING THE}

In a paper published earlier this year, cientists at Polymateria compared the precision and accuracy of several of these mining carbonyl index nal IR spectroscopy methods rely on
transmission of light through a material, bu this method is mostly outdated: it is only really applicable for thin films of plastic as larger chunks need to to enable testing which can be difficult for plastics made brittle by weathering.

Measuring the carbonyl index of a plastic requires comparing the peak another reference peak. When analysing thicker or more opaque materials using transmission spectroscopy the reference peak becomes saturated, giving an faccurate value for the carbonyl index. This can happen $0.1 \mathrm{~mm}$.

ATR-FTIR (Attenuated Total Reflection Fourier Transform Infra-Red) spectroscopy has largely superseded transmission IR spectroscopy. In ATR-FTIR, a crystal in conses light he sughe of the materia passes light through the surface of the detector. Almost no samak out to a 


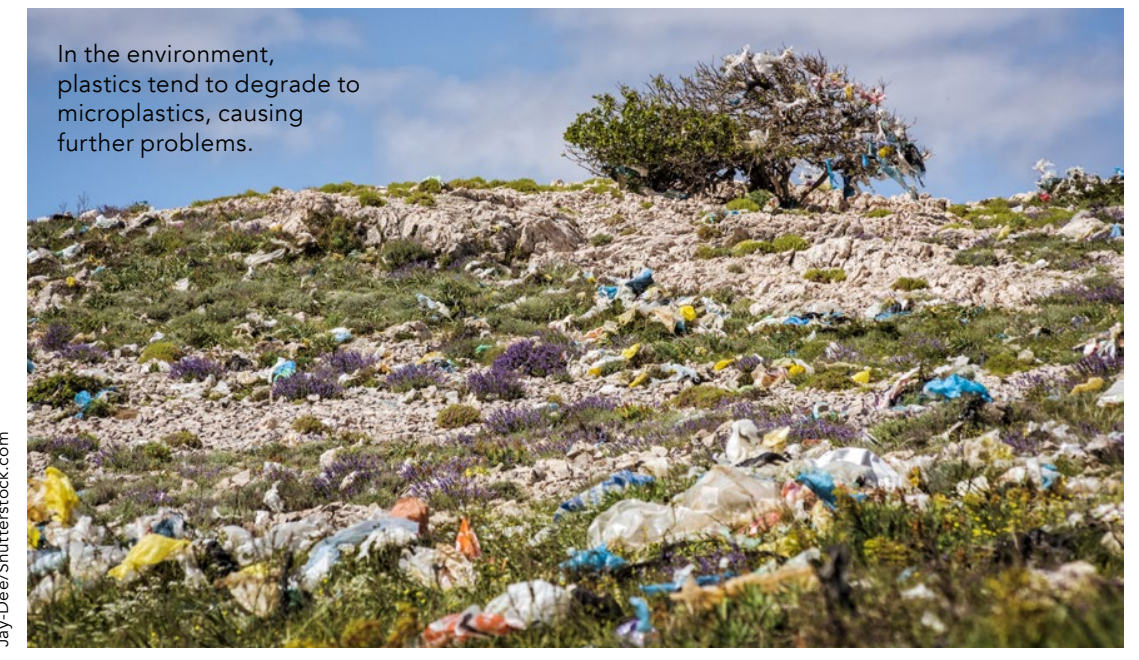

is needed, and samples can be analysed quickly at low cost.

There are multiple ATR-FTIR methods with different light angles and crysta types, which can affect the depth Problematically, many studies have. stated $\mathrm{Cl}$ values without speciffing the type of spectroscopy used, and some even describe using transmission IR spectroscopy despite testing materials up to half a millimetre thick - five times the desirable thickness, leading to $\mathrm{Cl}$ values being obtained of questionable validity

\section{LOOKING UNDER THE CURVE}

Having reviewed the common

spectroscopic methods, and performed their own tests, the Polymateria team proposed that the methodology for generating $\mathrm{Cl}$ standardised.

Their tests found

that the most

In Polymateria's approach being analysed and defines the area to the need for an operator to make an arbitrary decision as to which is the correct reference peak to analyse.

It also makes more intuitive sense than using peak heights. In infrared spectroscopy the height (or intensity) of of a chemical species. But in infrared spectroscopy very similar but chemicaly different species sit alongside, and even overlap, each other, in the same e of the spectra. This means that the height of the peak is not as informative as the combination of both height and breadh, i.e the area under the peak. The chemical change of a plastic sample SAUB method outperforms other stated methods of determining and can even be used to compare one test sample of plastic to another. They have also demonstrated that the method works for both polyethylene and polypropylene, allowing for comparisons to be made between these two different types of plastics.

The team also stress-tested their preferred method by analysing a plastic undergoing accelerated weathering Results for the SAUB showed better flexibility than other methods, increase reliability, and better precision for dentifying change over the course the plastic was highy ing when difficult to physically handle.

\section{PLASTIC ANALYSIS WE CAN TRUST}

The team at Polymateria were keen to

ensure that their discovery did not remain the intellectual preserve of academics. Despite successfully fulfilling the rigorous criteria of academic peer-review in publishing their work, they are now going a stage further in converting the SAUB methodology into a Standardised Test Method. Through a working group at the American Society of Testing and Materials (ASTM), the SAUB methodology is undergoing further technical scrutiny and review on its way to becoming an ASTM ternational Test peaks gives more accurate information on the goal is to the total concentration of CI species. give plastic

or determining consistent carbonyl Band (SAUB) method, a new way interpreting the same spectroscopic data from ATR-FTIR spectroscopy. Rather than taking the height of the carbonyl peak and comparing it to the height of the reference peak, the SAUB method takes the area under the carbonyl peak and compares it to the area under a universad eference peak for both polyethylene an polypropylene plastics.

This has some distinct advantages over conventional techniques: most the others, but the area of the peak gives the total concentration of all the sample, a far more useful indicator. Peak areas are therefore more reliably representative of what is ideally being measured in the spectroscopic process the concentration of all carbonyl species as an indicator of the extent of chemic change the plastic has undergone. Through their work, it became clear to Polymateria's team that th in the infrared area of the signals chemically similar species in the test specification, designed to determine the biodegradability of plastics in the case, the SAUB CI method provides one of the key indications in assessing he biodegradability performance of plastic, to verify claims made by plas biodegradable. In this BSI standard, the ey goal for achieving biodegradation is the chemical transformation of the plastic material into a bioavailable wax The SAUB CI measures the extent of this transformation and allows for a number to be assigned to the wax determining its $\mathrm{Cl}$. This, in combination with other analyses, determines if a bioavailable wax has successfully been produced. The key shift here is to move away from unsubstantiated claims and to produce numerical data on the performance of a plastic matenal in line wh International Standards.

\section{TRUSTING PLASTIC}

(T) STAY OR 'GO'

Pey

Ple

the global economy and our way of life,

its production will inevitably continue.

In the meantime, we need to improve waste management systems so more plastic is successfully recycled and more gets collected responsibly, so that as little plastic as possible ends up on land and in the ocean.

What scientists, like those at Polymateria, are doing to help reduce this problem is in for plastics, as well as new plastic materials. These materials need to be environmere durable (..e., resistant to envirnmental docay if ltesed) or more before $(i \mathrm{e}$, use the environment to help change themselves into biodegradele materials, if they become littered).

In both cases, new analytical tools will be necessary to prove the performance of these novel innovations. Methodologies that are accepted by the rigorously precise world of academia, and yet are also used actively by industry, can provide a clear pathway for bringing greater confidence to the general public that a plastic material will either be durable or decay. The SAUB CI method and the way it has been created, is a good example of this collaborative approach of bringing credible innovation to evaluate the types of plastic produr

\section{Poly: Behind \\ materia: the Research \\ Polymateria}

E: innovations@polymateria.com W: Wttps://www.polymateria.com/

Further information
- ASTM webpage on Working Group for Carbonyl Index Standard: https:///www. - Linkediln for C Wallis: https://www.linkedin.com/in/christopher-wallis-phd-mrsc- Mrinkedln for G Hill: https://www.linkedin.com/in/gavin-hill-a4233729/

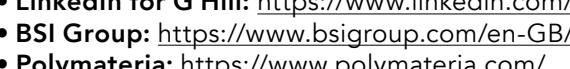
- Polymateria: attps:///www. polymateriac.com/ - Link to Paper on Identification of Marin Microm MIT/SUBCOMMIT/D20.htm - Link to Paper on Identification of Marine Microplastics: https: /
sciencedirect.com/science/article/abs/pi/S0025326X17310949

Research Objectives

Dr Christopher Wallis, Dr Gavin Hill and colleagues at Polymateria have been able to
standardise the method used to calculate the carbonyl index with important ramificton of chemical transformation undergone by a plastic material.

Detail

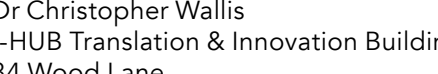

mperial College London White City

Campus
London

W12 OBZ

DrChristopher Wallis (MRSC,

Polymateria), leads the Research and Development team at the company's
London head-office. Dr Wallis' focus is the design, development and

testing of the company's bespoke
bio-transforming technology for plastic material, by both an internal interdisciplinary team and extensive

Funding
Polymateria Limited

Collaborators

- American Society for Testing and British Standards Institute (BS) - Dr Gavin Hill (Polymateria)

References

- Almond, J., ..., Wallis, C. (2020). Determination of the carbonyl index of polyethylene and polypropylene using specified area under band methodology
with ATR-FTR spectroscopy. e-Polymers. 20, 369-381, Wallis, C. (2019). Terrestrial fugitive plastic packaging: the blind spot in resolving
plastic pollution. Green materials., 1900044.

\section{Personal Response}

\section{Why do you think your team wat
in calculating carbonyl index?}

were the first to highlight this and make a huge effor Create a more definitive method, because we were interested not just in the $\mathrm{Cl}$ te that by combining the changes that may occur either to the height or the breadth of the peaks, we were obtaining far more accurate information, as the plastic sample chemically changed over time. This is critical to evaluating plastics in the natural
environment as the length of exposure they have is linked to the amount in which
they can be chemically ltered 